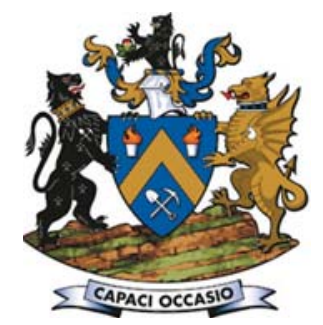

\title{
A modification of the zeta potential of copper sulphide by the application of a magnetic field in order to improve particle settling
}

\author{
by S. Gqebe*, M. Rodriguez-Pascual*, and A. Lewis*
}

\section{Synopsis}

Gravitational sedimentation of suspensions in various precipitation processes is hindered by colloidal stability. This is due to the high surface charge of the suspension and results in strong attraction/interaction between the ions on the particle surface and counter-ions in solution. Moreover, this strong interaction results in a charge build-up that renders the suspension stable. In order to induce gravitational sedimentation of these particles, a redistribution of ions close to the particle surface is required. We therefore sought to redistribute ions close to the particle surface by applying a magnetic field. This results in the reduction of interparticle electrostatic repulsive forces and a subsequent increase in the zeta potential of a suspension. For the purpose of this study, a copper sulphide suspension was used. Copper sulphide particles were exposed to a range of field strengths for set exposure times and their zeta potential was measured before and after exposure. All particles had an initial zeta potential value equal to or less than $-40 \mathrm{mV}$ prior to magnetic field exposure. A significant increase in zeta potential was observed, with values reaching a maximum of $-16.5 \mathrm{mV}$ when exposed to a $2 \mathrm{~T}$ field strength for 40 minutes. This is due to Lorentz ion shifts resulting from the Lorentz force exerted by the magnetic field on the particle surface.

\section{Keywords}

precipitation, sedimentation, copper sulphide, zeta potential, colloidal stability, electric double layer, charge destabilization.

\section{Introduction}

Metal sulphide precipitation is a process for treating industrial waste streams with high residual concentration of mixed metal ions and acid mine drainage. This process has been reported to achieve higher metal removal compared with the industrially prevalent lime precipitation, due to the sparingly soluble nature of the metal sulphide precipitates formed. However, precipitation is governed by high levels of supersaturation which favour the formation of fine particles (Lewis, 2010). Consequently, some of the metal sulphide fines formed exhibit slow settling dynamics and thus tend to remain suspended in solution.

The formation of fines, which reduces the efficiency of gravitational solid-liquid separation, is encountered in the mining industry. As a result, in most mining processes, large tanks to which chemical flocculant is added are required. Improving the settling characteristics of such fines without the addition of chemical flocculant would reduce both the settling time and volume required for settling. As a result, there is a need to investigate post-precipitation techniques that may improve the settling characteristics of such fines.

Commonly, fines suspended in solution are referred to as stable colloids. Colloidal stability may be attributed to strong interparticle electrostatic repulsive forces that hinder aggregation (Park et al., 2010).

When an electrolyte solution is in contact with another phase, such as a solid, gas, or another liquid immiscible with the electrolyte, a spontaneous redistribution of ions between the two phases takes place (Derjaguin, Churaev, and Muller, 1987). It follows that surfaces acquire a surface electric charge when brought into contact with a polar medium such as water. According to Derjaguin, Churaev, and Muller (1987) and Shiebl, Babick, and Stintz 2012), this charge may be acquired through surface ionization, ion adsorption, and ion dissolution.

The mechanism behind this acquisition of charge is as follows: a cloud of counter-ions forms around the charged particle in solution, creating an electro-neutral counter-ion complex (Haung et al., 1999; Velev and Bhatt, 2006). The ions in the fluid layer closest to the particle surface are strongly attracted to the substrate and are hence immobile. This is known as the Stern layer. Co-ions are repelled by the particle surface. However, they exist in the diffuse layer where both co-ions and counter-ions are found as mobile ions that are adsorbed in equilibrium with one another.

Collectively, the particle surface, Stern layer, and diffuse layer make up the electric double layer (Derjaguin, Churaev, and Muller, 1987). Within the electric double layer, the

* University of Cape Town, Cape Town, South Africa.

(C) The Southern African Institute of Mining and Metallurgy, 2016. ISSN 2225-6253. Paper received Nov. 2014; revised paper received Apr. 2015. 


\section{A modification of the zeta potential of copper sulphide}

concentration profile of ions gives rise to an electrostatic potential that decays exponentially with increasing distance from the charged particle surface. Along this electrostatic decay, various electrostatic potential points can be found that correspond to the surface, Stern, and zeta potentials.

In a colloidal dispersion, each particle possesses this electric double layer. It follows that the electric double layers of neighbouring particles interact with one another as a result of electrostatic and osmotic forces between the ions and the surfaces (Shiebl, Babick, and Stintz, 2012). This interaction results in changes in the ionic concentration and thus changes in the electrostatic potential distribution. These changes translate to a disturbance of the dissociation and adsorption equilibria within the diffuse layer and therefore change the surface distribution (Chan, 1976; Carnie, Chan, and Gunning, 1995; Chan et al., 2006). A strong overlap may even result in a change in the surface charge sign.

As a result of interacting double layers, an overall interaction energy between particles can be determined. This takes into account the interplay between the attractive van der Waals forces and the electrostatic repulsive forces between particles and can be used to determine whether the particles will attract or repel one another. The ionic concentration strongly affects the electric double layer. This is due to the Debye length, which is a characteristic of the electric double layer thickness and is inversely proportional to the square root of the ionic concentration of a solution. At high ionic strengths, colloids aggregate due to the screening of the charge on the colloids by the electric double layer. The converse is also true: at low electrolyte concentrations the colloids remain dispersed (Attard, 2001). This makes the ionic concentration a key parameter in determining the interaction energy between particles as the electric double layer is the sole contributor to the electrostatic repulsive force between particles. As a result, the balance between the van der Waals attractive force and the electric double layer repulsive force determines the stability of colloidal dispersions (Attard, 2001; Ohshima, 1995).

Colloidal stability is generally indicated by a high absolute magnitude of the zeta potential value, which is usually measured by micro-electrophoresis. This is in accordance with work conducted by Mokone, van Hille, and Lewis (2010) and Nduna, Rodriguez-Pascual, and Lewis (2013). By changing the zeta potential, one can influence the stability of a suspension and thus alter its settling characteristics. This zeta potential is defined as the potential difference between the particle surface and the bulk liquid (Velev and Bhatt, 2006). According to Lipus, Krope, and Crepinsek (2001) and Velev and Bhatt (2006) the zeta potential of a particle can be altered by the application of an external field. The reason for this is that when the colloids in solution are exposed to a magnetic field, a Lorentz force is created which acts on every charged particle moving through the field as shown in Equation [1]:

$$
F=q v \mathrm{~B}
$$

where $F$ is the Lorentz force $[\mathrm{N}], q$ is the electric charge [C], $v$ is the velocity $\left[\mathrm{m} \cdot \mathrm{s}^{-1}\right]$, and $B$ is the magnetic field strength [N.S. $\left.\mathrm{C}^{-1} \cdot \mathrm{m}^{-1}\right]$.

The Lorentz force effect on ions in the bulk of the solution can only be determined by Equation [1]. This
Lorentz force results in the shifting of ions both in the bulk solution and close to the particle surface (Lipus, Krope, and Crepinsek 2001). However, this ion shift is retarded by the liquid viscosity force. The viscosity force is equal to the Lorentz force in accordance with Newton's law for force balance. As a result, the Lorentz ion shift $\Delta x_{i}$ is derived from this relationship via Equations [1] and [2]:

$$
F_{v i s}=\frac{-6 \Pi \eta r_{i} \Delta x_{i}}{T}
$$

where $F_{v i s}$ is the liquid viscosity force [N], $\eta$ is the fluid viscosity $\left[\mathrm{kg} . \mathrm{s}^{-1} \cdot \mathrm{m}^{-1}\right], B$ is the magnetic field strength [N.s.C1.m-1], $t$ is the exposure time [s], $r_{i}$ is the ion radius [m], and $\Delta x_{i}$ is the Lorentz ion shift [m].

$$
\Delta x_{i}=\frac{q}{6 \pi \eta r_{i}} B t v
$$

Calculation of the Lorentz ion shift for ions in the bulk solution revealed that the Lorentz force has very little or no effect on the collision probability of these ions. However, the Lorentz ion shifts become significant close to the particle surface. Lipus, Krope, and Crepinsek (2001) reported that shifted counter-ions in the Stern layer will remain absorbed for a longer time according to desorption time estimations for bivalent ions of natural waters. This is because these bivalent ions have high desorption energies. Without strong adsorption of the shifted counter-ions in the Stern layer and electrostatic attractions, this desorption time would be much shorter. The ion shifts by Lorentz force translate into successful charge destabilization close to the particle surface and alter the zeta potential of particles.

Consequently, the focus of this research was to study the effect of a magnetic field on the interparticle electrostatic repulsive forces by measuring the zeta potential of a copper sulphide suspension.

Previous researchers showed that the application of a magnetic field to ferromagnetic particles, which are particles with a high magnetic susceptibility, increased the settled weight of these particles with time (Higashitani et al., 1994; Wang, Pugh, and Forssberg, 1994). Due to this susceptibility, ferromagnetic particles will strongly attract each other when exposed to a magnetic field as a result of applied magnetic forces. Wang, Pugh, and Forssberg (1994) observed an increase in the settled weight of chromite particles that were previously stabilized in solution with increasing magnetic field strength. In contrast, copper sulphide is diamagnetic and therefore has no magnetic properties. When copper sulphide particles are exposed to a magnetic field, aggregation is not achieved because of magnetic forces. Instead, the magnetic field exerts a Lorentz force on the particle surface and causes a redistribution of ions close to the particle surface, which results in the reduction in the interparticle electrostatic repulsive force. It is this reduction in the electrostatic repulsive force between particles that induces aggregation.

\section{Materials and methods}

There are two main steps involved in this study: (1) the precipitation of a copper sulphide suspension and (2) application of a magnetic field to this suspension. Experiments were conducted at atmospheric pressure and $23^{\circ} \mathrm{C} \pm 2^{\circ} \mathrm{C}$. A metal ion solution with a concentration of 


\section{A modification of the zeta potential of copper sulphide}

$500 \mathrm{mg} / \mathrm{L}$ was made by dissolving copper sulphate pentahydrate in de-ionized water and an equimolar sulphide solution was made by dissolving sodium sulphide nonahydrate in de-ionized water. Both solutions were stored in $4 \mathrm{~L}$ closed vessels as bulk solutions.

For a single experimental run, $500 \mathrm{~mL}$ of each solution was poured into separate beakers. $2 \mathrm{~mL}$ of sodium hydroxide was poured into the beaker containing the sodium sulphide solution in order to maintain the $\mathrm{pH}$ of the final copper sulphide suspension at a value above 6 . This was done in order to avoid the formation of hydrogen sulphide gas, which may result from the reaction at $\mathrm{pH}$ values below $6.2 \mathrm{~mL}$ of sodium hydroxide was also added to maintain a constant $\mathrm{pH}$ for each experimental run. The beakers were then sealed using parafilm. A $4 \mathrm{~mm}$ diameter hole was made in each parafilm layer in order to provide access via tubes to the solution inside each beaker. Nitrogen was sparged into each beaker for 20 minutes in order to minimize reactant oxidation. After sparging, the reaction proceeded in a Tmixing device supplied with reactants by a magnetic gear pump. For effective mixing in a T-mixer, a Reynolds number in the turbulent region must be maintained (Weigl, Bardell, and Cabrera, 2003). This corresponds to a pump speed equal to or greater than $1427 \mathrm{r} / \mathrm{min}$. Consequently, for each experimental run, a pump speed greater than $1427 \mathrm{r} / \mathrm{min}$ was used. Due to equipment limitations, a constant pump speed for each experimental could not be maintained and the speed varied between $1650 \mathrm{r} / \mathrm{min}$ and $2885 \mathrm{r} / \mathrm{min}$. A $1 \mathrm{~L}$ glass vessel was placed at the output end of the T-mixer to collect the formed copper sulphide suspension. From this vessel, a sample of the suspension was taken in order to measure the zeta potential immediately after precipitation. Figure 1 illustrates the set-up used for the precipitation step.

For sampling, a $10 \mathrm{~mL}$ syringe was filled with the copper sulphide suspension. This was then dispensed into a $42 \mathrm{~mL}$ sample bottle already containing $32 \mathrm{~mL}$ of sodium chloride spectator ions with an ionic strength of $0.01 \mathrm{M}$. The zeta

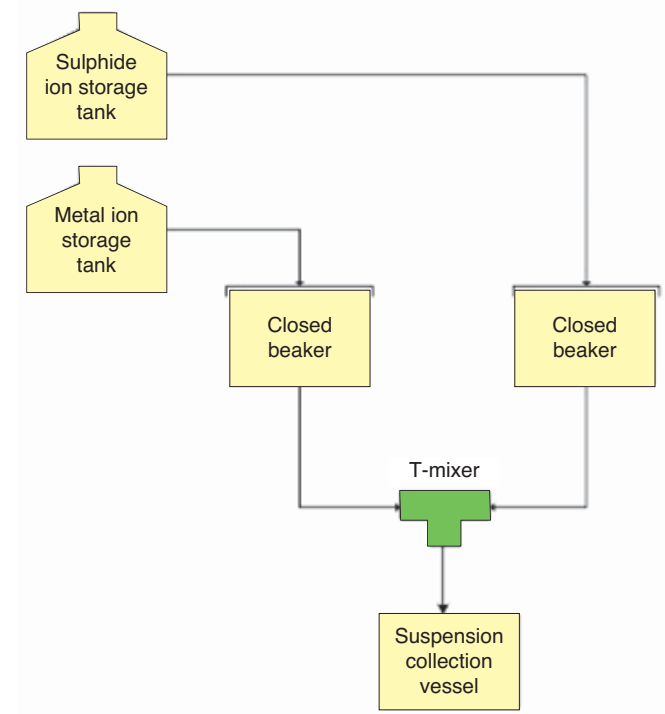

Figure 1-Experimental diagram for the precipitation of a copper sulphide suspension potential of the sample was then measured using a Malvern Zetasizer Nano. Once the zeta potential had been measured, the copper sulphide suspension was exposed to a magnetic field.

For this step, a cuvette with a volume of $2 \mathrm{~mL}$ was filled with the suspension. The cuvette was then placed in the middle of a $30 \mathrm{~cm}$ long stainless steel cylindrical sample holder with a diameter of $1.27 \mathrm{~cm}$. At this midpoint, the cuvette is exposed and not encased by the sample holder as there is a rectangular cutout. The sample holder was then placed between the two magnetic poles. This was a fixed position as the sample holder was screwed into the same position each time. Once the sample was in place, the magnet was switched on to the desired field strength for a set exposure time. For oscillating sample tests, the sample holder motor was switched on. The motor had a fixed speed of $2 \mathrm{~Hz}$. The field strengths and exposure times in Tables I and II were tested:

Subsequent to magnetic field exposure, the zeta potential of the suspension was measured once again.

\section{Results and discussion}

Each precipitated copper sulphide suspension had an initial zeta potential equal to or less than $-40 \mathrm{mV}$. Mokone, van Hille, and Lewis (2010) and Nduna, Rodriguez-Pascual, and Lewis (2013) reported that the zeta potential value of a copper sulphide suspension becomes more negative with increasing pH. Furthermore, Nduna, Rodriguez-Pascual, and Lewis (2013) attained a zeta potential value of $-50.1 \mathrm{mV}$ for

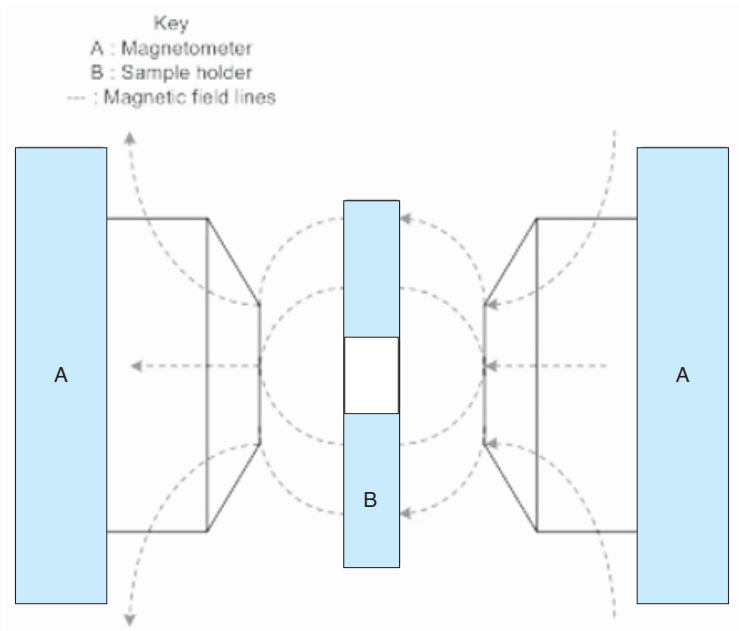

Figure 2-Schematic of magnetometer

Table I

First set of experiments conducted

\begin{tabular}{|l|c|c|}
\hline \multirow{2}{*}{ Sample type } & Magnetic field strength [T] & Exposure time [min] \\
\hline Non-oscillating sample & 0.5 & \multirow{2}{*}{40} \\
\cline { 2 - 2 } & 0.6 & \multirow{2}{*}{40} \\
\cline { 2 - 2 } & 0.7 \\
\cline { 2 - 2 } & 0.8 & \\
\cline { 2 - 2 } & 0.9 & \\
\cline { 2 - 2 } & 1 & \\
\end{tabular}




\section{A modification of the zeta potential of copper sulphide}

\begin{tabular}{|c|c|c|}
\hline \multicolumn{3}{|c|}{ Operating conditions for magnetic exposure } \\
\hline Sample type & Magnetic field strength $[T]$ & Exposure time [min] \\
\hline \multirow{9}{*}{ Non-oscillating sample } & \multirow[t]{3}{*}{1} & 10 \\
\hline & & 20 \\
\hline & & 40 \\
\hline & \multirow[t]{3}{*}{1.5} & 10 \\
\hline & & 20 \\
\hline & & 40 \\
\hline & \multirow[t]{3}{*}{2} & 10 \\
\hline & & 20 \\
\hline & & 40 \\
\hline \multirow[t]{9}{*}{ Oscillating sample } & \multirow[t]{3}{*}{1} & 10 \\
\hline & & 20 \\
\hline & & 40 \\
\hline & \multirow[t]{3}{*}{1.5} & 10 \\
\hline & & 20 \\
\hline & & 40 \\
\hline & \multirow[t]{3}{*}{2} & 10 \\
\hline & & 20 \\
\hline & & 40 \\
\hline
\end{tabular}

a copper sulphide suspension precipitated at $\mathrm{pH} 9$. Accordingly, this result was anticipated as each suspension was precipitated at a $\mathrm{pH}$ value of 6.3 .

Results from the first set of experiments listed in Table I revealed that a magnetic field had negligible effect on the change in the zeta potential of copper sulphide suspensions at field strength values less than $1 \mathrm{~T}$ at the exposure time used (Figure 3). At $1 \mathrm{~T}$, there is a noticeable change in the zeta potential of a copper sulphide suspension, from an initial value of $-40 \mathrm{mV}$ to $-36 \mathrm{mV}$. This resulted in a less negative zeta potential value. This increase in zeta potential translates to a decrease in the electrostatic repulsive force between colloids. However, the change in zeta potential observed at $1 \mathrm{~T}$ will not significantly increase the settling rate of the suspension. This is because a suspension with a zeta potential value of $-36 \mathrm{mV}$ is still within the stable colloid range, with a positive net interaction energy which indicates that repulsive electrostatic forces dominate.

A substantial change in the zeta potential of a copper sulphide suspension was seen at field strengths equal to or greater than $1 \mathrm{~T}$ when exposed for at least 40 minutes. As can be seen on Figure 4, at $1.5 \mathrm{~T}$, a negligible effect on particle zeta potential was observed for exposure times of 10 and 20 minutes. However, for an increased exposure time of 40 minutes, the zeta potential value of the copper sulphide suspension became less negative and increased from $-42.9 \mathrm{mV}$ to $-28 \mathrm{mV}$. The final zeta potential of this suspension is within the range for fast settling rates. This increase in zeta potential is due to a reduction in the interparticle repulsive forces, which favours aggregation as attractive forces now dominate.

At $2 \mathrm{~T}$, an insignificant effect on the zeta potential of a copper sulphide suspension was seen for an exposure time of 10 minutes. However, at this field strength, increasing the exposure time had an observable effect on the zeta potential of a copper sulphide suspension with a maximum of $-19.5 \mathrm{mV}$ being reached for an exposure time of 40 minutes. The zeta potential of this suspension increased from $-40 \mathrm{mV}$ to $-19.5 \mathrm{mV}$. A comparison of the DLVO plots in
Figure 5 for the initial and final zeta potential values for the 2 $\mathrm{T}$ suspension showed a decrease in the net interaction energy between colloids. This decrease is due to complete elimination of the electrostatic repulsive forces and thus an overall negative interaction energy with solely attractive van der Waals forces present. This favours aggregation and, by extension, improved settling rates.

The following equations were used to calculate the net interaction energy:

$$
V_{T}=V_{R}+V_{v d W}
$$

where $V_{T}$ is the net interaction energy [J], $V_{R}$ is the electrostatic repulsive force [J], and $V_{v d W}$ is the attractive van der Waals force [J].

$$
V_{R}=\varepsilon a \psi_{\mathrm{o}^{2}} \exp \frac{-\mathrm{r}(\mathrm{s}-2)}{s}
$$

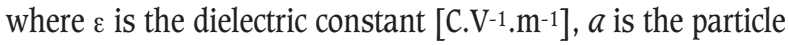
radius $[\mathrm{m}], \psi_{0}$ is the surface potential $[\mathrm{V}], r$ is interparticle distance $[\mathrm{m}]$, and $s$ is the normalized interparticle distance $[\mathrm{m}]$.

$$
V_{v d W}=-\frac{A}{6}\left[\frac{2}{s^{2}-4}+\frac{2}{s^{2}}+\ln \frac{s^{2}-4}{s^{2}}\right]
$$

where A is the Hamaker constant [J].

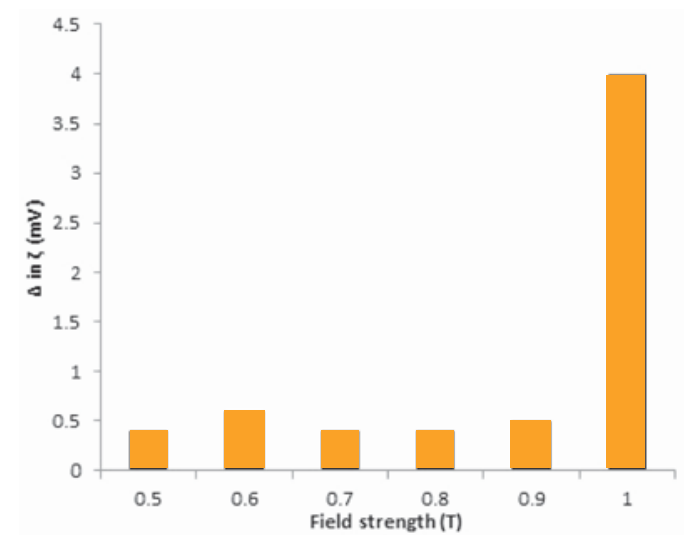

Figure 3-Change in zeta potential for a constant exposure time at various magnetic field strengths

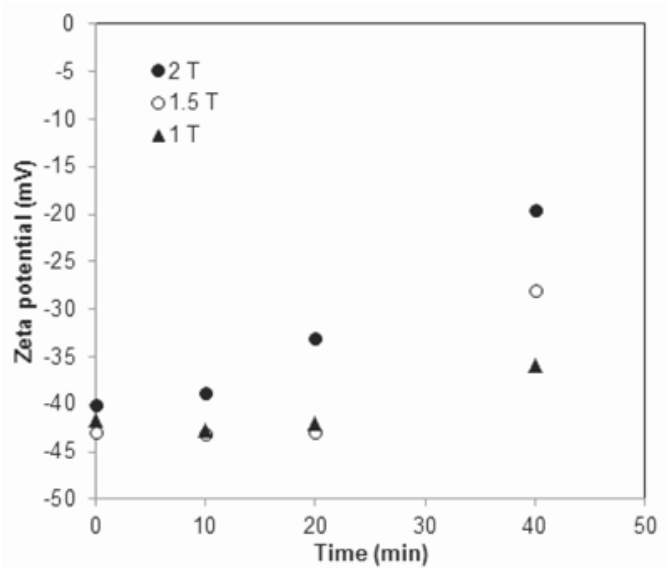

Figure 4-Zeta potential of copper sulphide as a function of exposure time at constant field strength 


\section{A modification of the zeta potential of copper sulphide}
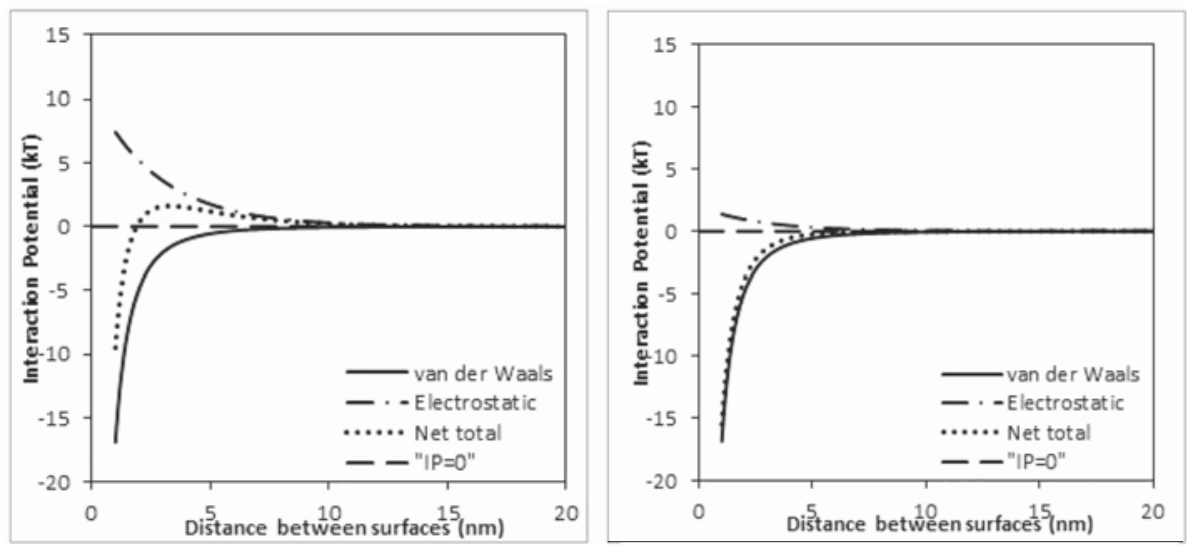

Figure 5-Net interaction energy plots for a suspension with an initial zeta potential of $-40 \mathrm{mV}$ (left) and final zeta potential of $-19.5 \mathrm{mV}$ (right)

Changes in zeta potential observed when suspensions are exposed to a magnetic field are due to the Lorentz force effect on ions close to the copper sulphide particle surface (Lipus, Krope, and Crepinse $\mathrm{k}, 2001)$. This effect is commonly known as the Lorentz ion shift and is solely responsible for the changes in zeta potential observed in this study.

According to Equation [1], the Lorentz force increases with increasing magnetic field strength at constant charge and particle velocity. This is why larger changes in the zeta potential of copper sulphide suspensions at higher field strengths are observed. It is imperative to note that although magnetic exposure destabilizes charge close to the particle surface, the overall system charge remains electro-neutral.

From the results obtained, it can be deduced that the application of a magnetic field improves the settleability of diamagnetic suspensions by eliminating interparticle electrostatic repulsive forces.

In the last set of tests (see Table II), copper sulphide particles were oscillated in a homogeneous magnetic field. Lipus, Krope, and Crepinsek (2001) reported that the most successful magnetic water treatment devices are of dynamic type, with an oscillating dispersion through a static magnetic field. It follows that the results obtained by oscillating copper sulphide in a homogeneous field showed improved results. A maximum zeta potential of $-16.5 \mathrm{mV}$ was obtained for an exposure time of 40 minutes. Zeta potential values of -22.3 $\mathrm{mV}$ and $-20.9 \mathrm{mV}$ were obtained for $1 \mathrm{~T}$ and $1.5 \mathrm{~T}$ respectively for a 40-minute exposure period.

A comparison of the results shown in Figures 4 and 6 reveals that the zeta potential of copper sulphide particles can be increased to within the range for fast settling rates at lower field strengths when the particles are oscillated in the magnetic field. This is because, for dynamic magnetic treatment, there is a significant reduction in the interparticle repulsive forces on each particle surface due to an increased Lorentz force. This results from an increase in the particle velocity and thus a greater Lorentz counter-ion ion shift in the Stern layer (Lipus, Krope, and Crepinsek, 2001). A comparison of Figures 4 and 6 also shows that an increase in field strength reduces the retention time required for increasing the zeta potential of a suspension to within the range for fast settling rates.

\section{Conclusions}

This study shows that there is significant potential in the application of a magnetic field to precipitation processes aimed at treating industrial waste streams with high residual metal ion concentrations, such as acid mine drainage, where the size and charge of the precipitates formed hinder gravitational solid-liquid separation. The technique used in this study not only improves the settling kinetics of a suspension, but does so without the addition of a chemical flocculant and therefore has an added advantage of reducing the volume required for settling. The zero additional chemical additives approach also reduces the environmental impact of industrial waste streams. This study has shown promising results on a laboratory scale; however, further investigation is required before it moves towards industrial application.

From the results, it can also be concluded that the zeta potential of a copper sulphide suspension may be modified by the application of a magnetic field. This is due to the ion redistribution on the particle surface as a result of the Lorentz force. However, this result is achievable only once a threshold magnetic field strength value of $1 \mathrm{~T}$ with a minimum exposure time of 40 minutes has been reached. In

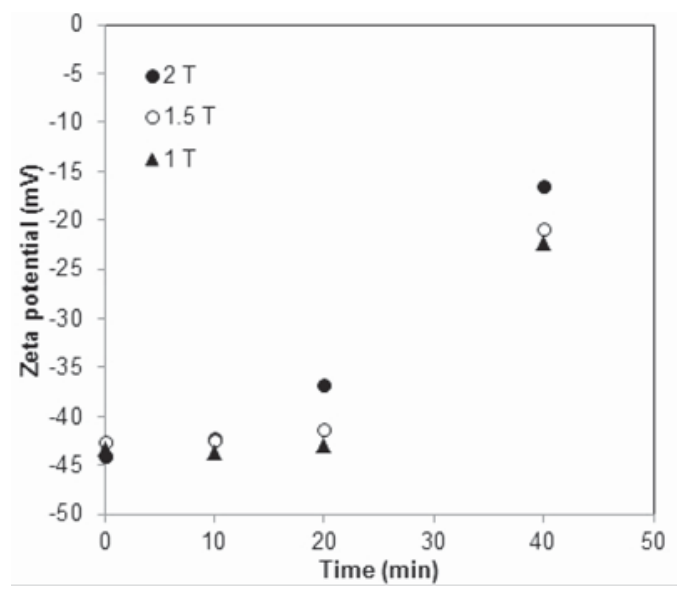

Figure 6-Zeta potential of copper sulphide as a function of exposure time at constant field strengths with a pulsating sample 


\section{A modification of the zeta potential of copper sulphide}

addition, it can be concluded that as the magnetic field strength is increased, a shorter exposure time is required to eliminate interparticle repulsive forces. This effect is amplified in the case where the suspension is oscillated in the magnetic field. As zeta potential is related to colloidal stability, a change in zeta potential can alter the settling characteristics of a suspension. An increase in zeta potential translates to a decrease in interparticle electrostatic repulsive forces and promotes aggregation. Consequently, shorter settling times are achieved.

\section{Recommendations}

Although the effect of a magnetic field on zeta potential has been shown, it is important to verify how long this effect lasts. It follows that determining the magnetic memory of the system is the first recommendation.

The effect of suspension speed on zeta potential could be tested only at a fixed speed of $2 \mathrm{~Hz}$ due to equipment limitations. Testing the effect of a range of speed values on zeta potential would provide a broad spectrum of results.

Although zeta potential may be used as a proxy to indicate the settleability of a suspension, further settleability tests may be done if there is interest in quantifying the settleability of the suspension.

The recommendations mentioned above can be achieved only with a magnet that possesses the following functions:

1. Multiple sample holder oscillating speed values

2. Increased sample volume for magnetic exposure

3. Higher magnetic field strengths.

Unfortunately, the magnetometer used in this study did not support these functions. As a result, these tests could not be conducted.

\section{References}

AtTARD, P. 2001. Recent advances in the electric double layer in colloid science. Current Opinion in Colloid and Interface Science, vol. 6, no. 4. pp. 366-371.

CARniE, S.L., Chan, D., and Gunning, J.S. 1995. Electric double layer between dissimilar spherical colloidal particles and between a sphere and a plate. Langmuir, vol. 10, no. 1. pp. 2993-3009

CHAN, D. 1976. Electrical double layer interactions under regulation by surface ionization equilibria--dissimilar amphoteric surfaces. Journal of the Royal Society of Chemistry, vol. 72, no. 1. pp. 2844-2865.

Chan, D., Healy, T.W., Supasiti, T., and Usui, S. 2006. Electrical double layer interactions between dissimilar oxide surfaces with charge regulation and Stern-Grahame layers. Journal of Colloid and Interface Science, vol. 296, no. 1. pp. $150-158$

Derjaguin, B.V., ChuRAev, N.V., and MulleR, V.M. 1987. Surface Forces. Consultants Bureau, New York.
Higashitani, K., Iseri, H., OKuhara, K., Kage, A., and Hatade, S. 1994. Magnetic effects on zeta potential and diffusivity of nonmagnetic colloidal particles. Journal of Colloid and Interface Science, vol. 172, no. 2. pp. 383-388.

Huang, Q.R., Dubin, P.L., Moorefield, C.N., and NewKome, G.R. 1999. Counterion binding on charged spheres: effect of $\mathrm{pH}$ and ionic strength on the mobility of carboxyl terminated dendrimers. Journal of Physical Chemistry B, vol. 104, no. 5. pp. 898-904.

LEwIS, A.E. 2010. Review of metal sulphide precipitation. Hydrometallurgy, vol. 104 , no. 2. pp. 222-234.

LiPus, L.C., KRoPe, J., and CREPinseK, L. 2001. Dispersion destabilization in magnetic water treatment. Journal of Colloid and Interface Science, vol. 236 , no. 1 . pp. $60-66$.

Мокоne, T.P., VAN HiLLE, R.P., and LewIS, A.E. 2010. Effect of solution chemistry on particle characteristics during metal sulfide precipitation. Journal of Colloid and Interface Science, vol. 351, no. 1. pp. 10-18.

Nduna, M., Rodriguez-PAscual, M., and Lewis, A.E. 2013. Effect of dissolved precipitating ions on the settling characteristics of copper sulphide. Journal of the Southern African Institute of Mining and Metallurgy. vol. 113, no. 5. pp. $435-439$

Oнsнiмa, H. 1995. Effective surface potential and double-layer interaction of colloidal particles. Journal of Colloid and Interface Science, vol. 174, no. 1. pp. $45-52$.

Park, Y., Huang, R., CoRTI, D.S., and Franses, I. 2010. Colloidal dispersion stability of unilamellar DPPC vesicles in aqueous electrolyte solutions and comparisons to predictions of the DLVO theory. Journal of Colloid and Interface Science, vol. 342, no. 2. pp. 300-310.

Shiebl, B., Babick, F., and Stintz, M. 2012. Calculation of double layer interaction between aggregates. Advanced Powder Technology, vol. 23, no. 2. pp. 139-147.

VelEv, O.D. and BHATT, K.H. 2006. On chip manipulation and assembly of colloidal particles by electric fields. Journal of the Royal Society of Chemistry, vol. 100, no. 27. pp. 38-750.

WANG, Y., Pugh, R.J., and Forssberg, E. 1994. The influence of interparticle surface forces on the coagulation of weakly magnetic mineral ultrafines in a magnetic field. Colloids and Surfaces, vol. 90, no. 2-3. pp. 117-133.

Weigl, B.H., BARDELL, R.L., and CABRERA, C.R. 2003. Lab on a chip for drug development. Advanced Drug Delivery Reviews, vol. 55, no. 3. pp. 349-377. 